\title{
Evidence-based Orthodontics: Has it something to do with your patient?
}

Fernanda Angelieri*

This section will be led in order to provide the clinician with support for comprehension and critical analysis of scientific articles, with elucidation of the main types of scientific studies, specific methodology and statistical analysis for clinical practice based in scientific evidence. The objective is to approximate clinician and science, easing the comprehension of the intricate scientific methods.

The scientific and technological innovation observed in the last decades in Orthodontics is undeniable. Obviously, we, orthodontists, want to offer the latest and most efficient treatment to our patients. However, how should our patients be submitted to these innovations? In other words, how should the orthodontist securely change the clinical conduct adopting new appliances, techniques or materials?

In recently published research, American orthodontists claimed to modify the treatment of their patients based, primarily, on the opinion of an expert, followed by consultation of published clinical cases, advice from colleagues and literature reviews, respectively. However, would it be wise to change the treatment of a patient based on the opinion of an expert?

In order to scientifically answer the clinical issues in Health, the concept of clinical practice based in scientific evidence initially appeared in Medicine, in the $90 \mathrm{~s}^{2}$ It consists in the search and applicability of the best and

How to cite this article: Angelieri, F. Evidence-based Orthodontics: Has it something to do with your patient? Dental Press J Orthod. 2013 Sept-Oct;18(5):11-3.

Submitted: July 29, 2013 - Revised and accepted: September 17, 2013.

*Associated Professor of the Methodist University of São Paulo. most current scientific evidence, in order to contribute on the decision making about the care in Health.,

Nowadays, facing the substantial offer of information, the process of search and identification of the best and most reliable scientific results, capable to guide the evidence-based clinical practice, comprises both hierarchy of scientific articles and the adequate interpretation of these results.

According to the hierarchy of scientific evidence, the systematic reviews and meta-analyses are found at the highest level of scientific evidence (Fig 1). Differently from the traditional literature reviews, in which the choice of scientific articles depended fundamentally on the authors, on systematic reviews the selection of scientific articles uses a rigorous scientific methodology, that must be understandable and reproducible. ${ }^{3}$ Moreover, a detailed analysis of the quality of the studies must be done, as well as an exhaustive search of all selected articles, according to strict inclusion and exclusion criteria, emphasizing the importance of the insertion of randomized clinical trials. When the results of these scientific studies selected on a systematic review can be grouped and statistically analyzed, this is a meta-analysis. Thus, the meta-analysis increases the general size of the sample and the power of the statistical tests, allowing better precision on the evaluation of the treatment effects. 


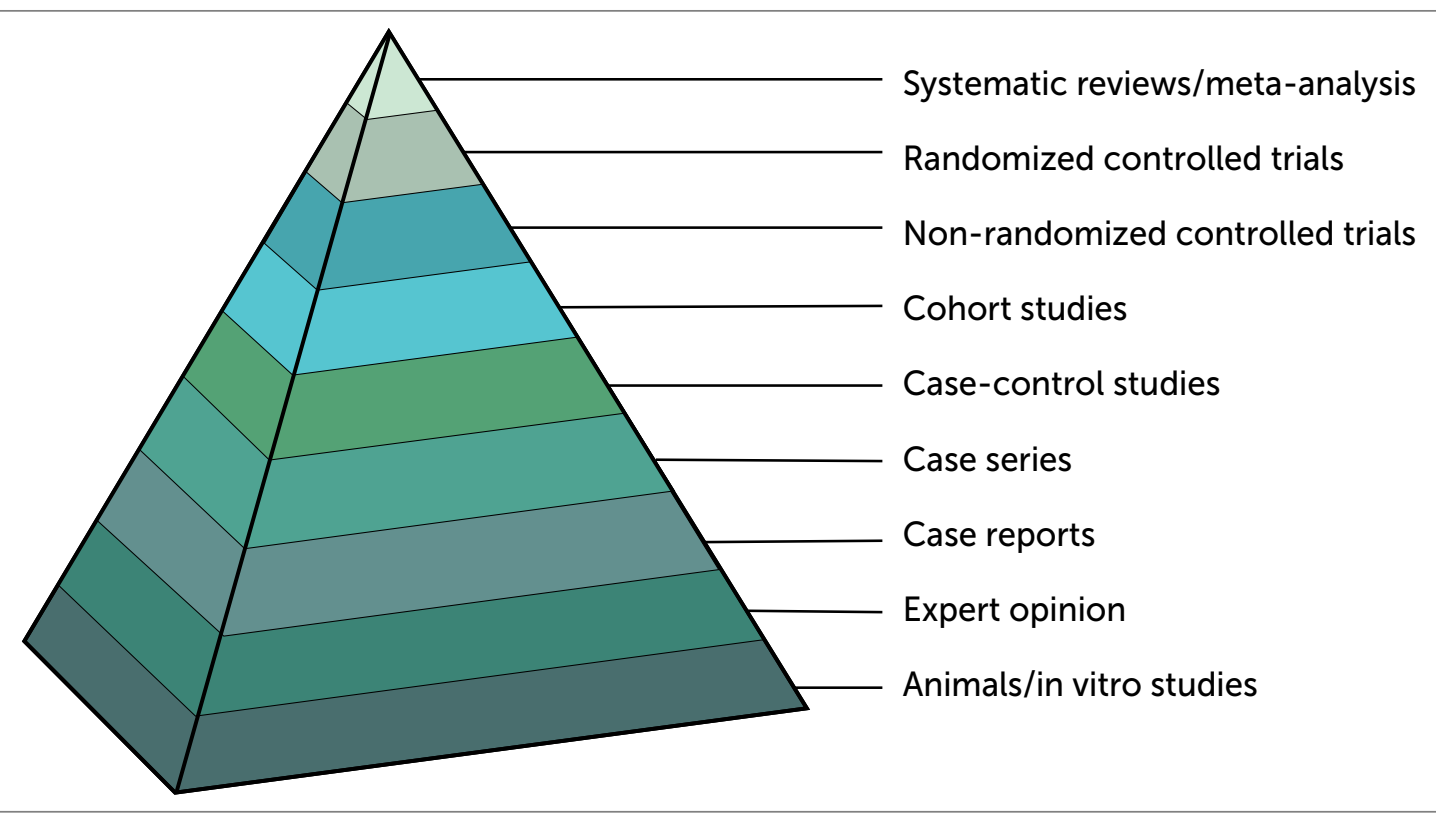

Figure 1 - Levels of scientific evidence in evidence-based Dentistry.

Additionally to the recent publication of several systematic reviews and meta-analysis in scientific orthodontic journals, there are non-profit scientific organizations designated to the production and distribution of systematic reviews in Health, such as The Cochrane Collaboration. These systematic reviews/meta-analysis can be obtained by accessing http://www.cochrane.org, in the Oral Health section.

The RCTs are considered the "gold standard" in therapeutic clinical studies. The randomization implies in random selection of individuals to compose the treatment and control groups, in addition to the random distribution of biases in the studied groups. ${ }^{3}$ Biases consist in any variable that may affect the study results. The more biases in the study,the smaller is the scientific value. However, many times, ethical and logistics issues involved in the selection and composition of the treated group and control group prevent the randomization of clinical trials. Despite the lower scientific value, the non-randomized trials provide important results on the evaluation of therapeutic issues in Health.

In a lower grade in the hierarchy of scientific evidence are the cohort studies. Generally, the cohort study refers to the natural history of a disease, to its prognosis or etiology. ${ }^{4}$ In this way, a group of indi- viduals is observed over time and a certain risk factor will be evaluated in relation to its evolution and influence on the pathology's natural course. For example, a study evaluating the incidence of temporomandibular disorders (TMD) in individuals subjected to stress (risk factor) and not stressed, with initial absence of signs and symptoms of TMD.

The case-control studies consist in the comparison between two samples. One will present the analyzed pathology or characteristic and the other will not. ${ }^{3}$ The correlation studies are considered case-control - for example, associating or not the lower inferior crowding with the presence or absence of lower third molars.

The case series - and, more specifically, the case reports - only demonstrate the ability of a single professional in treating one or more patients. Thus, this type of study not always reflects the reality of the use of a certain therapy when treating a patient randomly selected, ${ }^{3}$ considering the great amount of uncontrolled variables present.

Although deeply settled in orthodontic environment, the opinion of an expert assumes one of the lowest levels of scientific evidence. This is due to the highly subjective ${ }^{3}$ character implicit in the opinion, which generally includes the clinical experience of that professional, the acquired knowledge and 
personal and psychological experiences. However, we must remember that Orthodontics was built around great experts such as Angle, Tweed and Ricketts. Therefore, the opinion of an expert must be considered, but will only be valid as scientific evidence if proved by clinical trials, preferably randomized.

Despite the importance of studies in animals and in vitro, for certain topics of Orthodontics - such as, respectively, the evaluation of cytotoxicity and mutagenicity of materials used in Orthodontics or histological studies of orthodontic movement - these studies comprise the lowest level of scientific evidence for clinical practice. This is due to the fact that they do not refer directly to the clinical reality. ${ }^{4}$

Due to the great amount and diversity of scientific studies, it is worth emphasizing that the intention of organizing them hierarchically aims at obtaining fast and efficient clinical response based in scientific evidence, by means of consultation of systematic reviews/meta-analysis. However, despite the recent publication of several systematic reviews on The
Cochrane Collaboration and, specially, on scientific orthodontic journals, numerous clinical issues are still not totally answered by the systematic reviews/ meta-analysis. ${ }^{5}$

Given this current panorama, it is extremely important to search for all these types of studies on the main available databases, as: MEDLINE (publicly available at www.pubmed.org), LILACS (Latino American and Caribbean literature in Health sciences, available at http://lilacs.bvsalud.org/), SciELO (Scientific Electronic Library Online, available at www.scielo.br) and Portal Capes (available at www.periodicos.capes.gov.br). In addition, not only the identification of the type of study assumes emphasized relevance, but also the adequate interpretation of methodological quality of scientific articles. In the era of evidence-based clinical practice, it is extremely important that orthodontists have a greater understanding about the complex research design and statistical analysis to effectively treat their patients in the new millennium. ${ }^{6}$
REFERENCES

1. Madhavji A, Araujo EA, Kim KB, Buschang PH. Attitudes, awareness, and barriers toward evidence-based in orthodontics. Am J Orthod Dentofacial Orthop. 2011;140(3):309-16.

2. Sackett D, Rosenberg W, Gray J, Haynes R, Richardson W. Evidence-based Medicine: what it is and what it isn't. Br Med J. 1996:312(7023):71-2.

3. Ruiz M. Evidence-based orthodontics or the paradigm shift. Int Orthod 2011:9(1):1-19

4. Mariano MTS, Januzzi E, Grossmann E. Ortodontia baseada em evidência cientifica: incorporando ciência na prática clínica. Rev Dental Press Ortod Ortop Facial. 2009:14(3):107-13

5. Delière M, Yan-Vergnes W, Hamel O, Marchal-Sixou C, Vergnes J. Cochrane systematic reviews in orthodontics. Int Orthod. 2010;8(3):278-92.

6. Law SV, Chudasama D, Rinchuse D. Evidence-based orthodontics. Angle Orthod. 2010;80(5):952-6. 